\title{
MICROSTRUCTURE OF LATERALLY OVERGROWN GAN LAYERS
}

\section{Z. Liliental -Weber* and David Cherns \\ H.H. Wills Physics Laboratory, University of Bristol,Tyndall Avenue, Bristol BS* 1TL}

* on sabbatical leave from the Lawrence Berkeley National Laboratory, Materaials Science Division, Berkeley, CA 94720

\begin{abstract}
Transmission electron microscopy study of plan-view and cross-section samples of epitaxial laterally overgrown (ELOG) GaN samples is described. Two types of dislocation with the same type of Burgers vector but different line direction have been observed. It is shown that threading edge dislocations bend to form dislocation segments in the c-plane as a result of shear stresses developed in the wing material along the stripe direction. It is shown that migration of these dislocations involves both glide and climb. Propagation of threading parts over the wing area is an indication of high density of point defects present in the wing areas on the ELOG samples. This finding might shed new light on the optical properties of such samples.
\end{abstract}

\section{INTRODUCTION}

The use of highly lattice-mismatched substrates such as (0001) sapphire for the growth of nitride semiconductors has inevitably lead to high densities of threading dislocations in devices [12]. However, better device performances, especially for lasers, are usually achieved when the dislocation density is low. For this reason epitaxial lateral overgrowth (ELOG) was introduced [3-5] and this technique has resulted in a significant improvement in the performance of nitride laser diodes [6]. The ELOG approach consists of masking parts of the defective epilayer (GaN grown on $\mathrm{AL}_{2} \mathrm{O}_{3}$ or $\mathrm{SiC}$ ) with thin stripes of an amorphous layer (usually $\mathrm{SiO}_{2}$ ) so that dislocations under the mask are prevented from propagating into the overgrown parts of the layer during subsequent growth. Since GaN does not grow on the $\mathrm{SiO}_{2}$, continuation of growth takes place only on the seeds left uncovered by the amorphous material. As soon as growth front reaches the top of the mask, the GaN layer starts to grow not only vertically, along the c-axis, but also laterally, perpendicular to the c-axis, over the amorphous mask. Depending on the crystallographic direction of the mask stripes, 
the ratio of the stripe width to the stripe separation, and the growth conditions (temperature and III/V ratio) the lateral growth can be several times faster than the growth along the c-axis. During this growth step, dislocations close to the edge of the seed areas tend to bend to follow the faster growth direction, such that their line directions differ between the laterally grown parts (wings) and the seed columns. Ideally there should be no dislocations grown along the c-axis in the wing regions. However, since dislocation lines cannot finish inside bulk material (unless they combine with dislocations of opposite sign, i.e. forming a loop) some segments extending in the c direction will inevitably be formed, particularly at the meeting fronts of overgrown material. If new layers are subsequently grown, such dislocations will again propagate and reach active parts of the devices, usually limiting their performance.

Threading dislocations parallel to the c-axis are easy to detect using etching methods or/and scanning or atomic force microscopy. Dislocations with line directions close to c planes will not, however, be revealed using the above methods. Recent studies of the laterally overgrown layers with the $\mathrm{SiO}_{2}$ masks along [11 $\underline{2}$ 0] direction showed that c-axis pure edge dislocations with their Burgers vector $b=1 / 3<1120>$ can be completely eliminated, since they all bend onto the c-planes. However, mixed dislocations with Burgers vector $b=1 / 3<11 \underline{2} 3>$, which also bend onto the c-planes, have a greater tendency to propagate again to the sample surface [7]. However, the number of mixed dislocations which behave in this way is small compared to the total number of dislocations observed in the heteroepitaxial layers. This explains why the density of threading dislocations quoted in the literature $[3,8]$ is orders of magnitude lower $\left(10^{4}-10^{5}\right.$ dislocations $\left./ \mathrm{cm}^{2}\right)$ and $\left(6 \times 10^{7}\right.$ dislocations $\left./ \mathrm{cm}^{2}\right)$ [9] in the overgrown areas compared to the seed areas $\left(10^{9}-10^{10} \mathrm{~cm}^{-2}\right)$. The overall numbers of dislocations (both extended along the c-axis and on c-planes) should remain unchanged, but their distribution in the layer should be drastically different, much higher closer to the $\mathrm{SiO}_{2}$ masks and lower at the sample surface.

Due to difference in the thermal expansion coefficient between the underlying $\mathrm{SiO}_{2}$ masks and GaN seed areas and the likely presence of some impurities particularly on the seed edges, some additional mechanisms may play an important role, and the density of dislocations might be higher than expected. Observations by Sakai [10] indicate formation of small angle tilt boundaries at the wing/seed interfaces and also at the wing/wing meeting front (MF). However, this issue is disputed by different group of investigators [11]. Our earlier TEM studies of such samples showed that voids and high densities of dislocations are formed at the meeting fronts [12] as indicated by Sakai [13]. 
The contrast on TEM micrographs is different on the two sides of the meeting front indicating a relative misorientation [12]. Finite element calculation [14] shows that maximum stress gradient in the GaN occurs along the boundary between the tensile and compressive fields (GaN grown on GaN and $\mathrm{GaN}$ grown above $\mathrm{SiO}_{2}$ masks). This stress can be accommodated either by dislocation generation and/or dislocation propagation. Based on the mechanism described for GaAs grown on $\mathrm{Si}$, upward bending of the wings, as the result of different stress in the seed and wing area, was predicted and confirmed experimentally [15]. The same authors predicted that downward bending should be expected for GaN grown on $\mathrm{SiC}$ and this was indeed confirmed experimentally [16].

Very recent studies by Hacke et al [17] show the presence of nonluminescent defects at a $30^{\circ}$ angle to the [1 $\underline{100]}$ masking strip direction, e.g. parallel to the [2110] and [1210] directions. These are loops found to be screw dislocations with a short [0001] edge component connecting two parallel screws. The half loops are located above the seed with extension into the wing areas, but their origin was not determined. The authors [17] believe that defects present in their samples are different from these found by Sakai et al [13]. Hacke et al [17] associate the presence of these dislocation loops with the difference in thermal expansion coefficient between the substrate and the seed, and expect a force component to be present along the masking strip, which would lead to dislocation glide under the influence of a shear stress.

At present, the role of dislocations in accommodating thermal stresses, and the factors that control the final configurations of threading dislocations in ELOG material, are uncertain. In this paper, we examine the type and configuration of dislocations in the wing regions along with the lattice rotations present at the seed/wing and the wing/wing boundaries. Standard transmission electron microscopy (TEM) is used to provide information on the nature of these dislocations and their distribution. In order to provide full information, plan-view and cross-section samples have been studied, since, depending on the thickness of the grown layers, thinning of the samples from the substrate side for plan-view observation can provide information only from the top part of the layer. Relative rotations have been determined using the large angle convergent beam electron diffraction (LACBED) technique. By combining these results, it is proposed that threading dislocations are bent to form c-plane segments, as a result of shear stresses acting on the basal plane. The mechanism of migration, which involves both glide and climb, is discussed. 


\section{EXPERIMENTAL}

The sample studied was grown on an (0001) $\mathrm{Al}_{2} \mathrm{O}_{3}$ substrate by metal-organic-chemicalvapor-deposition (MOCVD), with an initial highly-dislocated GaN layer $2.75 \mu \mathrm{m}$ thick on which a $0.75 \mu \mathrm{m}$ thick $\mathrm{SiO}_{2}$ layer was deposited. By etching through the mask windows in the $\mathrm{SiO}_{2}$ layer with a width of $3.5 \mu \mathrm{m}$ were removed leaving trapezoidal strips of $\mathrm{SiO}_{2}$ aligned along the [1100] direction (Fig.1) with a $7 \mu \mathrm{m}$ width on the top of the trapezoid. After this procedure the GaN layer was grown a further $5 \mu \mathrm{m}$ thick above the mask to allow full coalescence of the overgrown areas. These studied layers were similar to those which were used for the first long-life lasers $[6,18]$.

Transmission electron microscopy was used to study the structure of the overgrown layers on these patterned substrates. Cross-section and plan-view thin films were prepared using typical methods of thin film preparation by dimpling, followed by ion milling in order to obtain electron transparency. Plan view samples were thinned from the substrate side and cross-sectioned samples were first cut with a width of about $1 \mathrm{~mm}$ and glued together face to face with additional supporting sapphire layers on both sides of the ELOG samples. Two different microscopes were used for this study: an 002B Topcon microscope with an accelerating voltage of $200 \mathrm{KeV}$, and a Philips 430 operated at $250 \mathrm{KeV}$ with sample tilting up to about $\pm 60^{\circ}$ which was particularly useful for g.b analysis to determine dislocation Burgers vector and Large Angle Convergent Beam Electron Diffraction (LACBED) for tilt/twist determination between wing/wing meeting front (MF) and wing/seed areas.

\section{RESULTS AND DISCUSSION}

\section{III.1. Plan-view Samples}

TEM studies of plan-view samples show the dislocation arrangements in the top half-micron of the GaN. Fig. 2 shows the different arrangement of dislocations above the seed areas where GaN was grown over $\mathrm{GaN}$ deposited on $\mathrm{Al}_{2} \mathrm{O}_{3}$, and in the wing areas where $\mathrm{GaN}$ was grown over $\mathrm{SiO}_{2}$ deposited on $\mathrm{GaN} / \mathrm{Al}_{2} \mathrm{O}_{3}$. In the seed areas, dislocations are end-on arranged on grain boundaries (see inset in Fig. 2), similar to those present in uniform $\mathrm{GaN}$ films grown over $\mathrm{Al}_{2} \mathrm{O}_{3}$ or over $\mathrm{SiC}$ [19]. Their density in the seed areas is in the range of $1 \times 10^{8} \mathrm{~cm}^{-2}$. At the boundaries between the

seed and wing areas, there are arrays of dislocations which run approximately along the stripe direction. Some of these dislocations bend out into the wing region. At the wing/wing meeting 
fronts (MFs), similar in-plane dislocations along the stripe direction are present with segments close to the boundary which bend in the form of half-loops into the wing regions. Some asymmetries in the distribution of these half loops is observed. This is most visible at the borders between the seed and the wing. There are areas where only few dislocations can be observed, but in some other areas the number of dislocations increases s see the left (L) and the right (R) interface with the seeds-Fig. $2\}$.

For the half loops which emanate from the seed/wing interface, such half loops consist of three parts -two threading parts (marked by T on Figs. 3 and 4) on the two ends of the half loop arranged in directions close to the c-axis and the main part which lies close to the c-plane, such that their lines are easily observed in plan-view samples (Fig. 3). The threading parts at the seed/wing interface are not easy to show on the same micrograph where the half loop is observed, since the dislocation line extends for a long distance along this interface and only later bends to the wing area. Such threading parts at the other end of dislocation loops can, however, be seen in Fig.4 together with dislocation extending to the wing. The second threading part is in the wing area (Fig.3-also indicated by $\mathrm{T}$ for each half loop). These threading parts end at the substrate side of the TEM foil and may start at the $\mathrm{SiO}_{2}$ layer. The distance between threading dislocations at the seed/wing interface (Fig. 4) is on average $0.5 \mu \mathrm{m}$, but varies locally in the range $0.35-2.25 \mu \mathrm{m}$. The threading parts located at the border between the seed and the wing areas are of edge character.

Half loops which are at the boundary between two wings consist also out of three parts with a main part on the c-plane and two threading parts, one at the wing/wing boundary and the second part in the wing area (Fig.5). Fig. 6 shows some half loops at both sides of the wing/wing boundary, one can notice that the loops are elongated along the stripe direction [1100]. Burgers vector analysis of the half loops shows that they are of mixed character with $b=[1210]$ or $[\underline{2} 110]$ at $30^{\circ}$ to the stripe direction, and with their leading parts close to pure edge orientation. This suggests that the driving force for their formation is a shear stress acting on the (0001) plane and in the stripe direction. The loops on opposite sides of the boundary bow in opposite directions but have the same Burgers vector, which means they are responding to opposite stresses.

One can also notice that these half loops start from threading parts either from the seed/wing interface (Figs.3 and 4) or at the wing/wing interface (Figs. 5 and 6), then propagate parallel to the stripe direction, depart from this interface and subsequently propagate to the opposite interface. However, if obstacles such as treading dislocations are present, these half loops can interact with 
each other forming a different type of dislocation (Fig.7). For clarification, the arrangement of dislocations e.g. half loops with their threading parts at either seed/wing or wing/wing MFs are schematically shown on Fig. 8. It is also possible that threading dislocations present in the wing areas are also sources for half loop formation. Such a half loop would propagate to the nearest interfaces, e.g. either wing/wing meeting front or seed/wing interface.

Quite often a difference in contrast on the two sides of the wing/wing interface can be noticed on TEM micrographs, especially when two beam conditions are applied (see Fig.1). This suggests that these two wing areas are misoriented to each other. This is also in agreement with the fact that the density of dislocations at the interface with the GaN seed areas is often higher than in the seed area. Burgers vector analysis showed that many of these boundary dislocations had Burgers vectors of $1 / 3[11 \underline{2} 0]$ type, i.e. perpendicular to the trace of the boundary. To check the misorientation, LACBED patterns with convergence angles of about $6^{0}$ were taken for the regions encompassing the wing/wing boundary. In Fig 9, the sample was oriented to include the (1010) systematic row of reflections. Besides the (1010) contours, other contours inclined about $10^{0}$ (marked as A) and about $25^{\circ}$ (marked as B) to the (1010) contours are also visible. The measurements based on the shift of diffraction lines across the meeting front (Fig.9) shows that

these two wing areas are misoriented on average by $0.07^{0}$ perpendicular to the wing/wing boundary and by $0.11^{\circ}$ and $0.14^{\circ}$ on inclined directions A and B, respectively, indicating that both tilt and twist are present at this boundary.

\section{III.2. Cross-section samples}

Studies of cross-section samples confirm our observations on plan-view samples. A high dislocation density is observed at the interface between the seed and the wing area and their line directions are almost parallel to each other (see Fig. 10). Other dislocation lines are visible in the wing region close to the seed. When the sample is tilted such that the c-planes are inclined to the vertical, these dislocations appear curved in projection (Fig. 10a). However, when the c-planes are tilted to edge-on, these dislocations, as well as most of the dislocations in the wing/seed boundary appear straight, parallel to the c-plane. Under these conditions, the dislocations show a residual dotted contrast. By examining the contrast of these dislocations under various two-beam conditions it was confirmed that the Burgers vectors were of $1 / 3<1120>$ type. Thus the residual contrast in Fig 
$10 \mathrm{c}$ is consistent with $\mathbf{g} \cdot \mathbf{b}=0$ while the oscillations can be ascribed to depth variations of the dislocations in the foil (Fig. 10c). This is consistent with the arrangement of $1 / 3<11 \underline{2} 0>$ dislocations in plan-view configuration, which for a long distance are almost parallel to the seed stripe and then start to depart by formation of half loops (see Figs. 1 and 3-7), which occasionally reach the wing/wing boundary (Fig. 7).

High dislocation densities are also observed at the wing/wing boundary (Figs. 5-7). These dislocations are of the same type, and therefore can be treated as grain boundary dislocations. This grain boundary is not a perfect one, appearing as a kind of triple boundary, since three main sets of dislocations can be observed (Fig. 11). The distance between the two outer sets of dislocations (two grain boundaries) was about $0.5 \mu \mathrm{m}$ close to the sample surface. It was noticed that close to the $\mathrm{SiO}_{2}$ only one boundary was present. The estimated density of dislocations at the central boundary at this interface was about $2 \times 10^{10} \mathrm{~cm}^{-2}$. This number would increase by adding the dislocations from the outer interfaces, as one can see on (Fig. 11-a). Again this shows that the dislocation density at this boundary is higher than expected, since, if only bending dislocations were present, this number should not be significantly higher than the density of dislocations at the seed area, around $2 \times 10^{8} \mathrm{~cm}^{-}$

2 . In some areas nanotubes along the c-axis were formed in the vicinity of the outer boundaries (see inset in Fig. 11b).

It was not easy to observe in our cross-section samples the threading parts aligned along the c-axis which terminate the half-loops, as observed close to the top surface in plan-view configuration. Occasionally one can see such dislocations near vertical orientation (Fig. 11). There are many inclined dislocations and some dislocations align along c-axis just at the upper interface with the $\mathrm{SiO}_{2}$ strip (see inset in Fig. 11a). This "discrepancy" between cross-section and plan-view samples is not surprising since the distance between the threading parts is rather large (a few micrometers), and, therefore, not easy to observe in the cross-section thin foil. The other point is that the threading parts are slightly inclined to c direction and this may explain why only some parts of them are visible for a particular cross-sectioned sample.

LACBED patterns were taken to explain rotations at the seed/wing and wing/wing boundaries. To investigate twists, the sample was oriented to include $\mathrm{g}=0002$ systematic row reflections in different areas of the cross-section sample, e.g., at the left (L-Fig.1) and at the right (R-Fig.1) interfaces between the seed and ELOG area and at the meeting fronts between two wing areas (MF-Fig.1). To investigate tilts, LACBED patterns with $\mathrm{g}=[11 \underline{2} 0]$ systematic row reflections 
were also examined. At the left interface (L) the 0002 contours were shifted (Fig. 12a) by an angle which varied from area to area between $0.02^{0}$ to $0.07^{0}$. This is consistent with a twist about the interface normal. When the [1210] contours were examined, the rotation angle was larger, around $0.3^{0}$, consistent with a tilt axis along the stripe direction. For the wing/wing boundary, this angle for the (0002) planes, measuring twist, varied between $0.09^{0}$ to $0.12^{0}$ (Fig. 12b). Much larger rotations, corresponding to tilts, $0.15^{\circ}$ and $0.26^{0}$ was measured for the (1120) contours. For the right interface (R) between the wing and the seed areas the twist angle measured from the shift of 0002 Kikuchi lines is slightly larger then on the left interface, and it is between $0.1^{0}$ to $0.12^{0}$. Almost no tilt was noticed for the perpendicular direction at this measured interface.

\section{III.3. Discussion}

Our observations of plan-view and cross-section samples show that the ELOG method leads indeed to lower densities of dislocations with line direction close to c-axis. However, within the wing areas, i.e. the overgrown GaN, we find a low density of threading dislocations $\left[1.4 \times 10^{7} \mathrm{~cm}^{-2}\right]$ along with $\left[2 \times 10^{7} \mathrm{~cm}^{-2}\right]$ dislocations of $1 / 3<1120>$ type, with Burgers vectors at $30^{0}$ to the stripe direction (type A dislocations), bowed in the form of half-loops in the (0001) plane. These defects appear to originate at both the seed/wing and wing/wing interfaces and extend either to a terminating threading dislocation in the wing area or propagate to the opposite interface (Figs. 3, 5-7 and schematic on Fig. 8). In contrast, the seed/wing and wing/wing boundaries also contain many dislocations of $1 / 3<1120>$ type elongated along stripe direction with Burgers vectors at $90^{0}$ to the stripe direction (type B dislocations), which do not bow into the wing regions (Figs. 3-7 and schematic on Fig. 8).

LACBED studies show that there are rotations at both the seed/wing and wing/wing boundaries. Although there is some variation, especially at the interfaces with seeds, we find twists of typically up to $0.1^{\circ}$, and significantly larger tilts, up to $0.3^{\circ}$. The latter is consistent with the high density of type B dislocations, with a $0.3^{\circ}$ tilt corresponding to a dislocation separation of about $0.1 \mu \mathrm{m}$. This is close to the measured spacing $0.08 \mu \mathrm{m}$ in Fig. 4 at the seed/wing interface and $0.06 \mu \mathrm{m}$ in Fig. 5 at the wing/wing interface. Where dislocations of type B are present in the boundary planes, they represent a tilt corresponding to the edge component of the Burgers vector. For the A dislocations on the other hand, we expect a twist due to the screw parts. A twist of $0.1^{\circ}$ 
would correspond to a spacing of about $0.3 \mu \mathrm{m}$, or 1-2 such dislocations in a typical area of the plan-view foil. This is not inconsistent with the observations [Fig.6].

The presence of dislocations at the wing/wing and seed/wing boundaries might be partly due to misorientations in the seed material that inevitably result from the columnar growth of GaN. However, we believe the type A dislocations are introduced in response to shear stresses which act down the [1100] strip direction. This is the direction in which tensile stresses will be developed, either during or after growth due to the mismatch in thermal expansion coefficients between GaN and the $\mathrm{SiO}_{2}$. Where buckling occurs, shear as well as tensile stresses should also occur. We might expect these to vary along the stripes if the bond between the $\mathrm{SiO}_{2}$ and $\mathrm{GaN}$ is locally broken. This is consistent with the observation of the $\mathrm{SiO}_{2} / \mathrm{GaN}$ interface where many small voids are present with a larger void at the wing/wing MF (note inset in Fig. 1). With a shear stress along the stripe, we expect a Peach-Koehler force to act on dislocations of type A, but not on those of type B. Since the Peach-Koehler force acts everywhere perpendicular to the dislocation line direction we expect dislocations of type A to form half-loops as observed (see Figs. 3 and 5-7). Some elongation in the near screw direction may be explained by the greater line energy of the edge as opposed to the screw parts of the loop. Where a shear stress is initiated in one wing region, relaxation may occur in opposite senses across the wing/wing boundary, as observed in Fig. 5, where two half-loops of the same Burgers vector propagate in opposite directions on two sides of the wing/wing MF. Variations in the shear stress along the stripes will cause variations in the numbers of A dislocations as also observed. Note that the number of A dislocations on opposite sides of the wing/wing MF is larger than in the central part of the Fig. 4. The opposite situation one can observe on Fig. 4 (seed/wing interface) where in the center of the figure the number of A dislocations is much larger than in the upper part, where some interaction between dislocations took place.

Whereas the bowing of the A type dislocations in the (0001) plane is a glide process, the threading parts at the end of these half-loops will propagate by climb. Since climb requires motion of point defects, we expect the threading segments to trail the leading parts of the A dislocations. This can be observed on Fig. 6 and its inset where the outer half -loop has a threading part at the wing/seed interface and the second at the seed/wing interface. This half-loop interacts with a dislocation which is out of contrast on the left inset. A similar situation can be also observed on Fig. 7 (lower part) where two half loops propagating from the opposite interfaces interact with some obstacle forming a new dislocation. The form of the B dislocations is not unlike that described by 
Matthews and Blakeslee [20], for the propagation of misfit dislocations with the proviso that the Matthews-Blakeslee mechanism while operating in response to shear stresses, is a wholly glide process, with the threading segment leading rather than trailing growth.

\section{SUMMARY}

TEM studies of plan-view and cross-section samples have shown the arrangement and type of dislocations formed in the laterally overgrown GaN samples. Our samples differ from other samples described in the literature in that the ELOG layers were grown on reasonably low dislocation density GaN samples $\left(1 \times 10^{8} \mathrm{~cm}^{-2}\right)$. In these samples, the density of threading dislocations (and comparable density of laterally arranged dislocations) decreased by one order of magnitude $\left(1.4 \times 10^{7} \mathrm{~cm}^{-2}\right)$, slightly smaller than reported by Chen $\left(6 \times 10^{7} \mathrm{~cm}^{-2}\right)$ [9] and much higher than reported by Zhelewa $\left(10^{4}-10^{5} \mathrm{~cm}^{-2}\right)[8,14,16]$. Our observations reveal two types of dislocation with segments in the basal plane, edge dislocations with Burgers vectors 1/3[1120] (type B dislocations) lying in the wing/seed and wing/wing boundaries, and those with Burgers vectors of $1 / 3[\underline{2} 110]$ and 1/3[1210] (type A dislocations). We propose that type A dislocations are generated by shear stresses operating parallel to the stripe direction; type B dislocations should be unaffected by these stresses.

The A type dislocations, however, appear to be different than these observed by Hacke et al [17] since in our samples only one part of the half-loop was at $30^{\circ}$ to the stripe direction and the other part of this half-loop was not parallel to the first one. The bowing of this dislocation is due to glide under the action of the Peach-Koehler force which acts perpendicular to the dislocation line. Fig. 13 illustrates this mechanism schematically. Based on our observations the half-loops observed here may have started from the threading parts present on the interfaces (see schematic on Fig. 8), or possibly in the wing regions, but were never present in the seed areas. The threading parts must propagate by climb. This suggests that there is a high density of point defects in this area allowing this climb. This may allow us to understand the work of Chichibu [21], who found no substantial difference in optical properties for InGaN quantum wells grown on ELOG and non-ELOG substrates, if we assume that photoluminescence decay time is dominated by these point defects rather than extended defects.

Type B dislocations are reasonable to consider as grain boundary dislocations. Their arrangement can be seen clearly on cross-section micrographs (Figs. 10 and 11). These results are 
consistent with a previous study by Sakai [10], despite that in our samples the measured tilt is approximately by one order of magnitude lower (a tilt of about $0.1^{\circ}$ and a twist $0.3^{\circ}$ ) than this indicated in [10] or [11] of about $1^{\circ}$. This is an important finding which indicates that if any tilt/twist occurs at the seed/wing or wing/wing MFs new dislocations need to be formed in order to accommodate this misorientation. We believe that this misorientation is mainly introduced by a compressive/tensile stress at the seed/wing interfaces but also by columnar growth of GaN in the seed area. This would be consistent with our observation that the tilt/twist slightly varies depending on measured area of a particular interface and this would lead to a local difference in a density of dislocations present there (Fig. 1). In agreement with the prediction by Zytkiewicz [15] and experimentally studies of Zhelewa [16], we find that the overgrown regions tilt down towards the substrate.

In summary our study showed the presence of two types of dislocations (half-loops A and those elongated along the stripe direction B) formed in wing areas of ELOG samples. Threading dislocations were present on both ends of half-loops. Glide process lead to bowing of part of dislocations. The separation of the c-axis segments, called here threading parts, by climb is a strong indication of a high density of point defects present in the wing areas [21].

\section{ACKNOWLEDGMENT}

This work was partly supported by Air Force Office of Scientific Research under Order No. AFOSR-ISSA-00-0011 obtained through U.S. Department of Energy and partly by the UK Engineering and Physical Sciences Research Council (EPSRC) under grant GR/N16426. Z L W wants to thanks Drs. M. Benamara and J. Jasinski for the preparation of the micrographs for the publication and W. Swider for the excellent TEM sample preparation. 
Figure Captions:

Fig. 1. Cross-section TEM micrograph of the investigated ELOG sample. Overgrown seed and wing areas are shown: L-left interface at the seed/wing area, R-right interface the wing/seed area, MFmeeting front of two wing areas. Note high density of threading dislocations in the seed areas, dislocations at the MF, and different contrast on the both sides of the MF. The inset shows the interface roughness and formation of small voids at the $\mathrm{SiO}_{2}$ interface.

Fig. 2. Plan-view micrograph of the overgrown GaN layer; L, R, and MF as in Fig. 1. Note high density of dislocations (slightly arcing) in the wing areas on the $\mathrm{L}$ and $\mathrm{R}$ interface. Dislocations parallel to the stripe direction are present at the MF. The inset shows edge-on dislocations in the seed area arranged on grain boundaries (the inset is taken in different diffraction condition than the main micrograph in order to see defects in this area).

Fig. 3. Propagation of half-loop dislocations in the wing area from the interface with the seed. Threading parts ending these half-loops are marked by T. Note high density of dislocations at the wing/seed and wing/wing MF parallel to the stripe direction and the same inclination angle of threading parts in the wing and the seed area.

Fig. 4. Arrangement of dislocations at the different parts of the sample. Starting from the left: edgeon dislocations at the seed area, dislocations at the seed/wing interface along the stripe direction and threading parts marked by $\mathrm{T}$. At the right -a dislocation in the wing area departing from the seed/wing interface with its threading part $\mathrm{T}$ at this interface. The extension of this dislocation propagating through the wing area is shown on Fig. 6 (left side).

Fig. 5. Half-loops formed at the wing/wing MF. Note that two main half-loops propagate in the opposite direction on the both side of this interface, however on the right side propagation of the half-loop in opposite direction on the same side of the interface is also observed. Note presence of the threading parts at the wing/wing interface. 
Fig. 6. (a) Half-loops at the wing/wing interface. Note interaction of the main half loop with another dislocations. The later dislocation is out of contrast in (b) and (c) shows presence of two dislocations in this area.

Fig. 7. Half-loops propagating to the opposite interfaces. Note that this propagation can be interrupted (lower part of the micrograph) by defects present in the wing area.

Fig. 8 Schematic showing an arrangement of dislocation half-loops observed in plan-view samples, where two types of dislocations, A-half loops and B-dislocations along a stripe direction, are formed with their threading parts $(\mathrm{T})$ propagating through the wing area. Eventually such a threading part can reach an opposite interface-showed by dotted line.

Fig. 9. LACBED pattern obtained for [1010] reflection. Note a shift of the $<10 \underline{10}>$ lines and inclined A (A') and B (B') lines when they intersect the wing/wing MF.

Fig. 10. Dislocations in the wing area (both bending from the seed/wing interface and propagating from this interface). Note change of their contrast with a change of the diffraction vector. Dotted contrast of these dislocations present in (c) is due to arcing of these dislocations in the c-plane.

Fig. 11. Cross-section view of the wing/wing MF in two different diffraction condition. Note a high dislocation density at this interface (a) and a triple boundary in (b). On the right hand side a long dislocation which has a strong c-component is visible which might be a threading part connected with a half-loops formed on c-plane. Note many inclined dislocations in the wing areas, which also might be threading dislocations, connected with half-loops. A high density of such inclined dislocations are present at the interface with the $\mathrm{SiO}_{2}$ (see inset in a). Note formation of a nanotube on the outer boundary at the meeting front (inset in b).

Fig. 12.(a) LACBED patterns for $\mathrm{g}=0002$ observed at the seed/wing interface (a) and wing/wing MF (b). Note a shift of the main and inclined lines indicating tilt and twist present at these interfaces. 
Fig. 13. Schematic diagram to illustrate the growth of the half-loops under the action of glide forces due to a shear stress acting in the wing region (bounded by horizontal lines) along the stripe direction. It is envisaged that a threading dislocation bends to form a segment in the basal plane. Once initiated, the glide force acting perpendicular to the dislocation line at all points, causes the basal plane segment to bow into a half-loop, limited by the extent of the wing region. The original threading segments (dashed lines) can only move by climb, which is slow, providing a limit on the extent of half-loop propagation

\section{REFERENCES:}

1. Z. Liliental-Weber, S. Ruvimov, W. Swider, Y. Kim. J. Washburn, S. Nakamura, R.S. Kern, Y. Chen, and J.W. Yang, Mat. Res. Soc. Proc. 482, 375 (1998).

2. F. Ponce, D.P. Bour, W. Gotz, and P.J. Wright, Appl. Phys. Lett. 68, 57 (1996).

3. T.S. Zheleva, O.H. Nam, M.D. Bremser, and R.F. Davis, Appl. Phys. Lett. 71, 2473 (1997).

4. D. Kaplonek, S. Keller, R. Ventury, R.D. Underwood, P. Kozodoy, S.P. Den Baars, and U.K. Mishra, Appl. Phys. Lett. 71, 1204 (1997).

5. J. Park, P.A. Grudowski, C.J. Eitingm and R.D. Dupuis, Appl. Phys. Lett. 73, 333 (1998).

6. S. Nakamura, M. Senoh, S. Nagahama, N, Iwasa, T. Yamada, T. Matsushita, H. Kikyoku, Y. Sugimoto, T. Kozaki, H. Umemoto, M. Sano, and K.Chocho, Jap. J. Appl. Phys., Part 3, 36, L1568 1997.

7. A. Sakai, H. Sunakawa, A. Kimura, and A. Usui, J. Electron Microscopy, 49 (2), 323 (2000).

8. T.S. Zhelewa, O.H. Nam, J.D. Griffin, M.D. Bremser, and R.F. Davis, Mat. Res. Soc. Symp. Proc. 482, 393 (1997).

9. Y. Chen, R. Schneider, S.Y. Wang, R.S. Kern, C.H. Chen, and C.P. Kuo, Appl. Phys. Lett., 75, 2062 (1999).

10. A. Sakai, H. Sunakawa, and A. Utsui, Appl. Phys. Lettr., 73, 481 (1998).

11. P. Fini, L. Zhao, B. Moran, M. Hansen, H. Marchand, J.P. Ibbetson, S.P. DenBaars, U.K. Mishra, and J.S. Speck, Appl. Phys. Lett., 75, 1706 (1999).

12. Z. Liliental-Weber, M. Benamara, W. Swider, J. Washburn, J. Park, P.A. Grudowski, C.J. Eiting, and R.D. Dupuis, MRS Internet J. Nitride Semicond. Res., 4S1, G4.6 (1999).

13. A. Sakai, H. Sunakawa, and A. Utsui, Appl. Phys. Lett., 71, 2259 (1997).

14. T.S. Zhelewa, W.M. Ashamawi, O,-H. Nam, and R.F. Davis, Appl. Phys. Lett. 74, 2492 (1999).

15. Z.R. Zytkiewicz and J. Domagala, Appl. Phys. lett. 75, 2749 (1999).

16. T.S. Zhelewa, S.A. Smith, D.B. Thomson, K.J. Lithicum, P. Rajagopal, and R.F. Davis, J. Electon. Mater. 28, L5 (1999).

17. P.Hacke, K. Domen, A. Kuramata, T. Tanahaski, and O. Ueda, Appl. Phys. Lett. 76, 2547 (2000).

18. S. Nakamura, M. Senoh, S. Nagahama, N. Iwasa, T. Yamada, T. Matsushita, H. Kikyoku, Y. Sugimoto, T. Kozaki, H. Umemoto, M. Sano, and K. Chocho, Jpn. J. Appl. Phys. Part 2 36, L1568 (1997). 
19. Z. Liliental-Weber, S. Ruvimov, T. Suski, J.W. Ager III, W. Swider, Y. Chen, C. Kisielowski, J. Washburn, I. Akasaki, H. Amano, C. Kuo, and W. Imler, Mat. Res. Soc. Symp. 423, 487 (1996). 20. J.W. Matthews and A.E. Blakeslee, J. Cryst. Growth 27, 118 (1974).

21. S.F. Chichibu, H. Marchand, M.S. Minsky, S. Keller, P.T. Fini, J.P. Ibbeyson, S.B. Fleischer, J.S. Speck, J.E. Bowers, E. Hu, U.K. Mishra, and S.P. DenBaars, Appl. Phys. Lett. 74, 1460 (1999). 


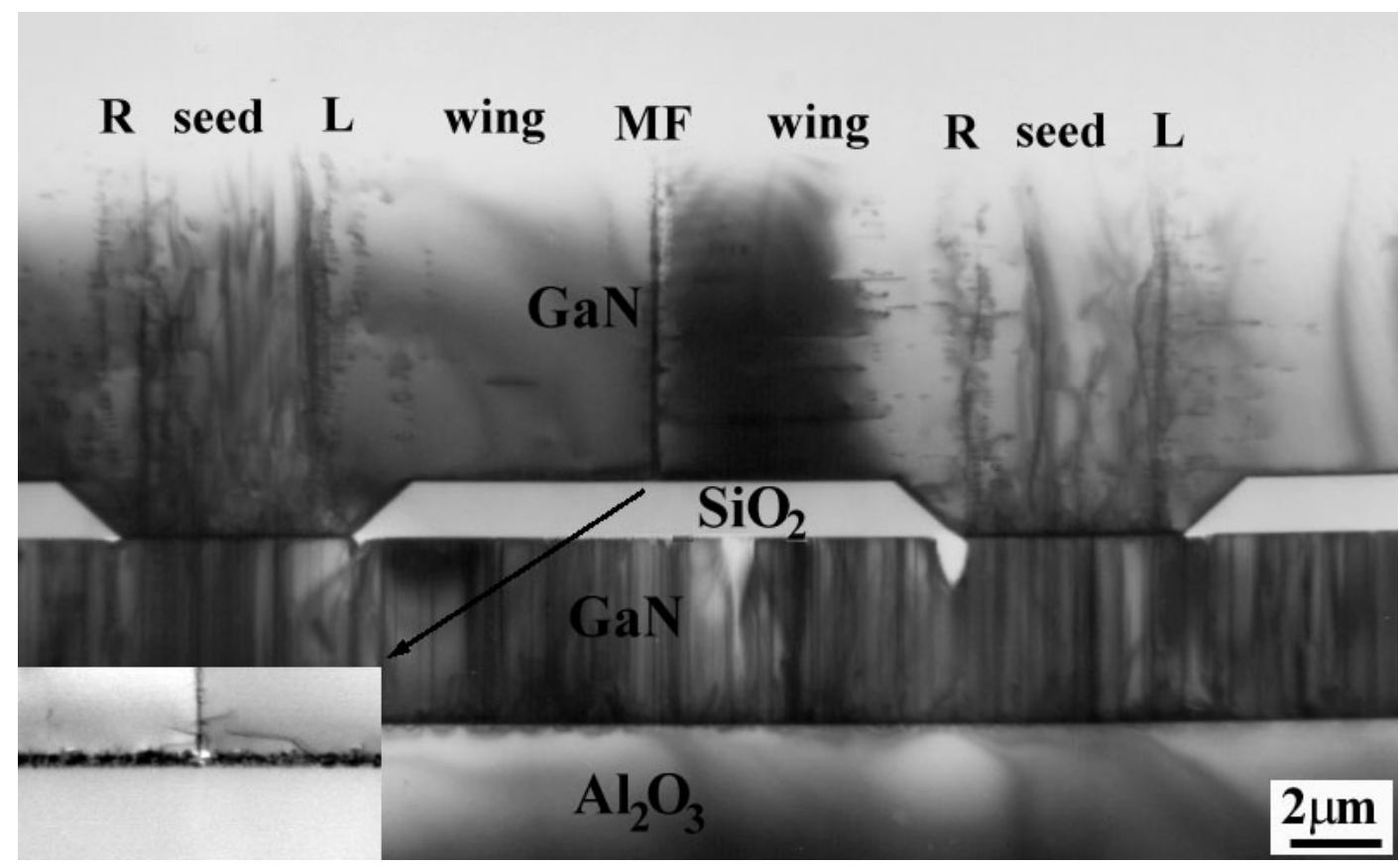

Fig.1. Z. Liliental-Weber and D. Cherns 


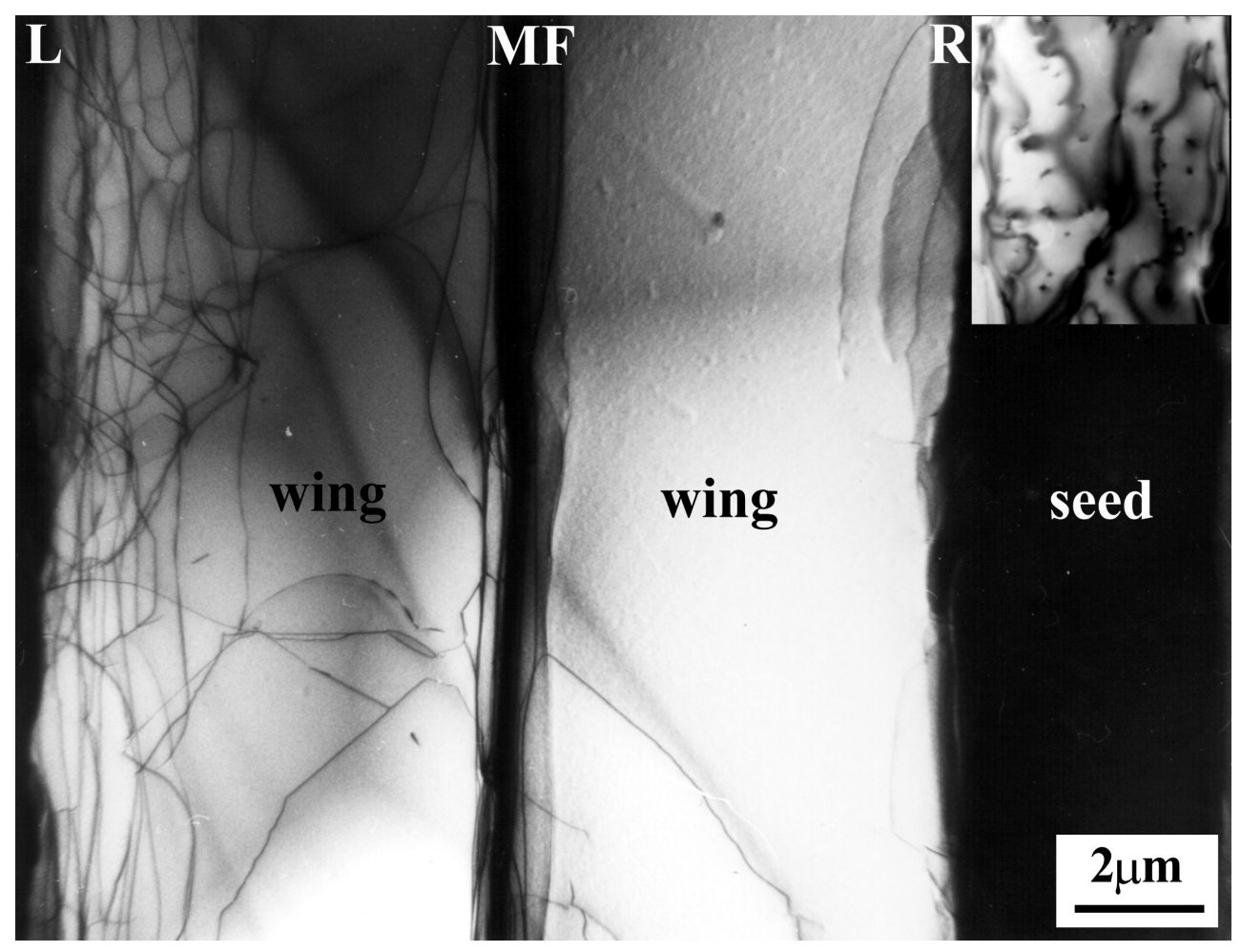

Fig.2. Z. Liliental-Weber and D. Cherns 


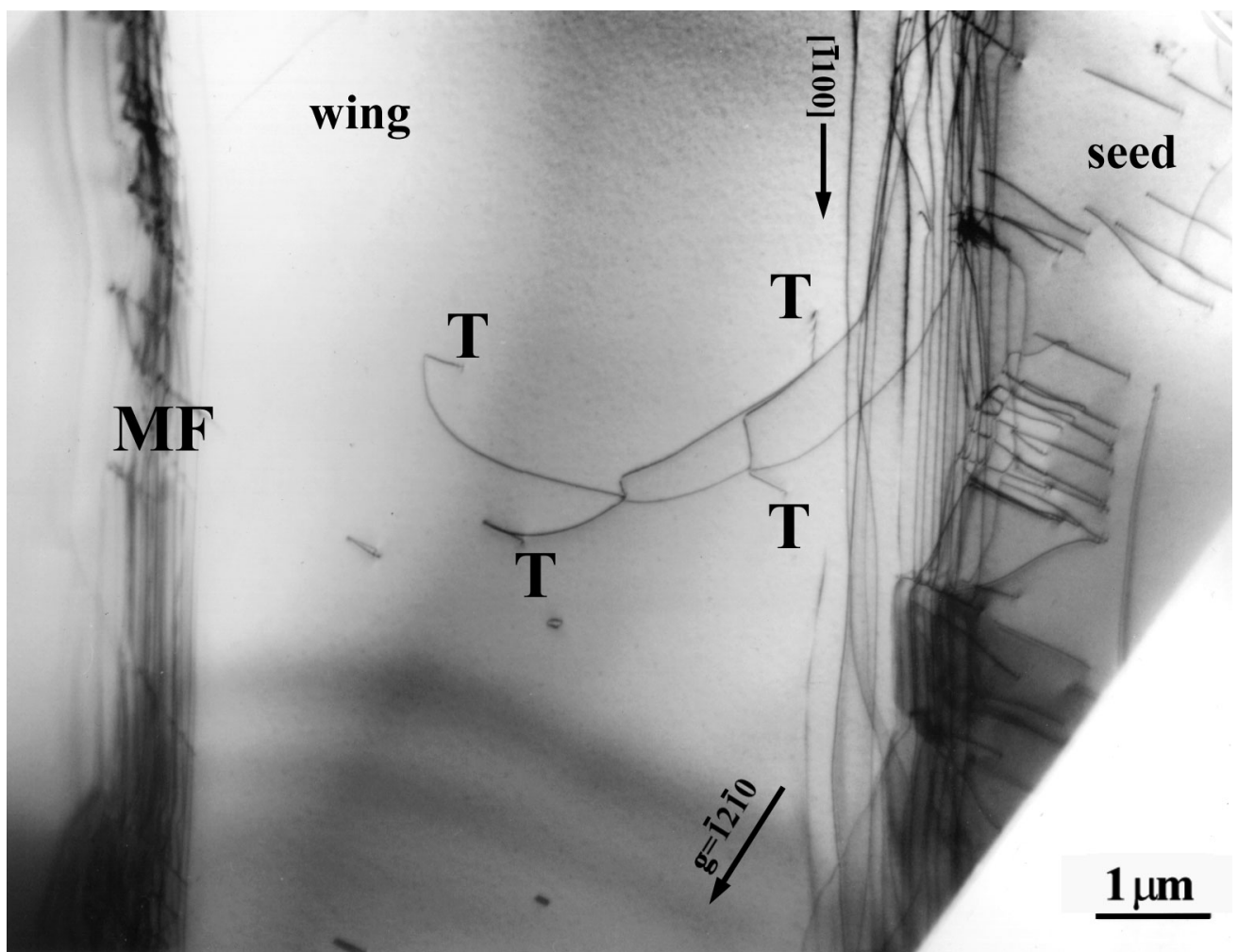

Fig.3. Z. Liliental-Weber and D. Cherns 


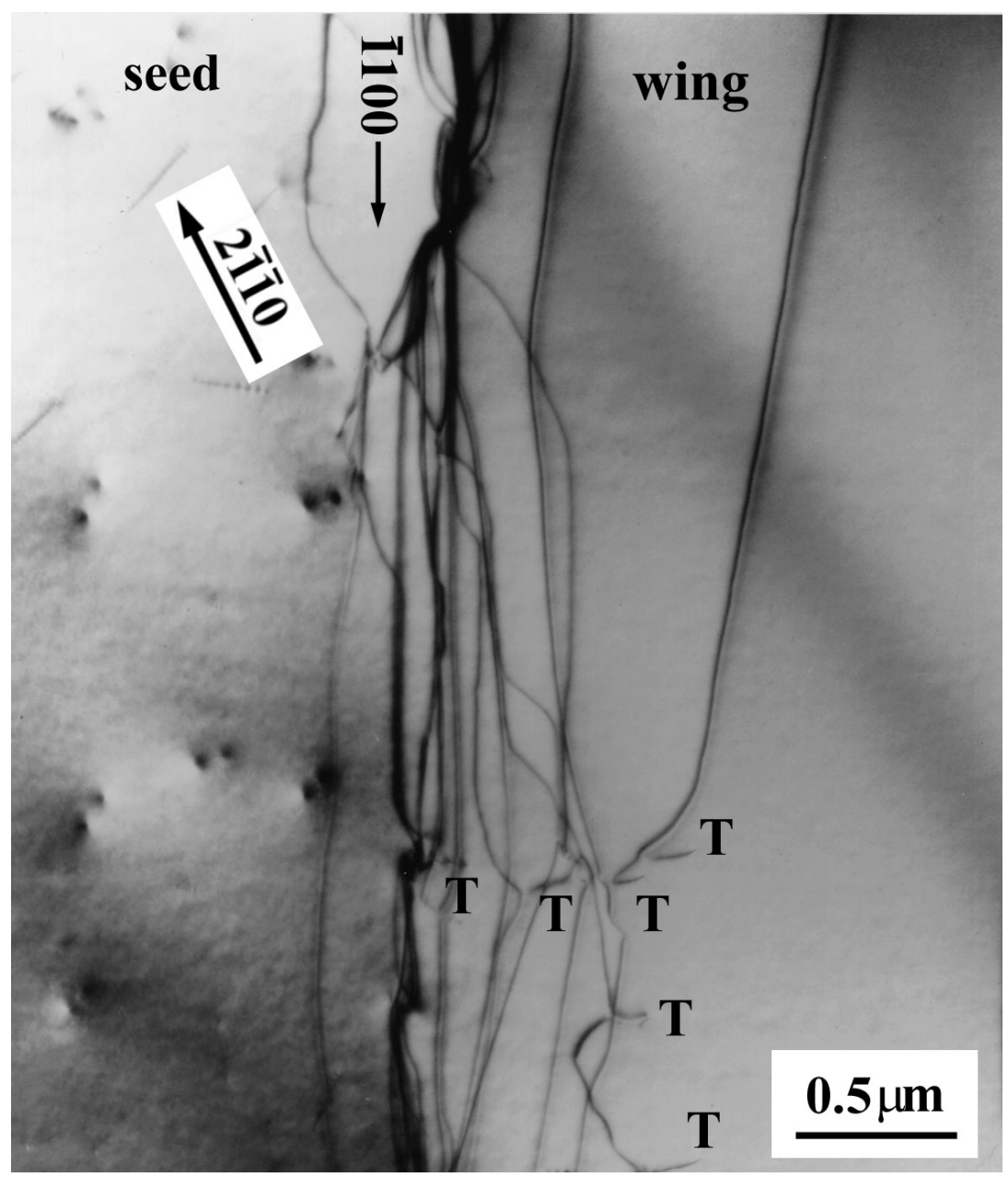

Fig.4. Z. Liliental-Weber and D. Cherns 


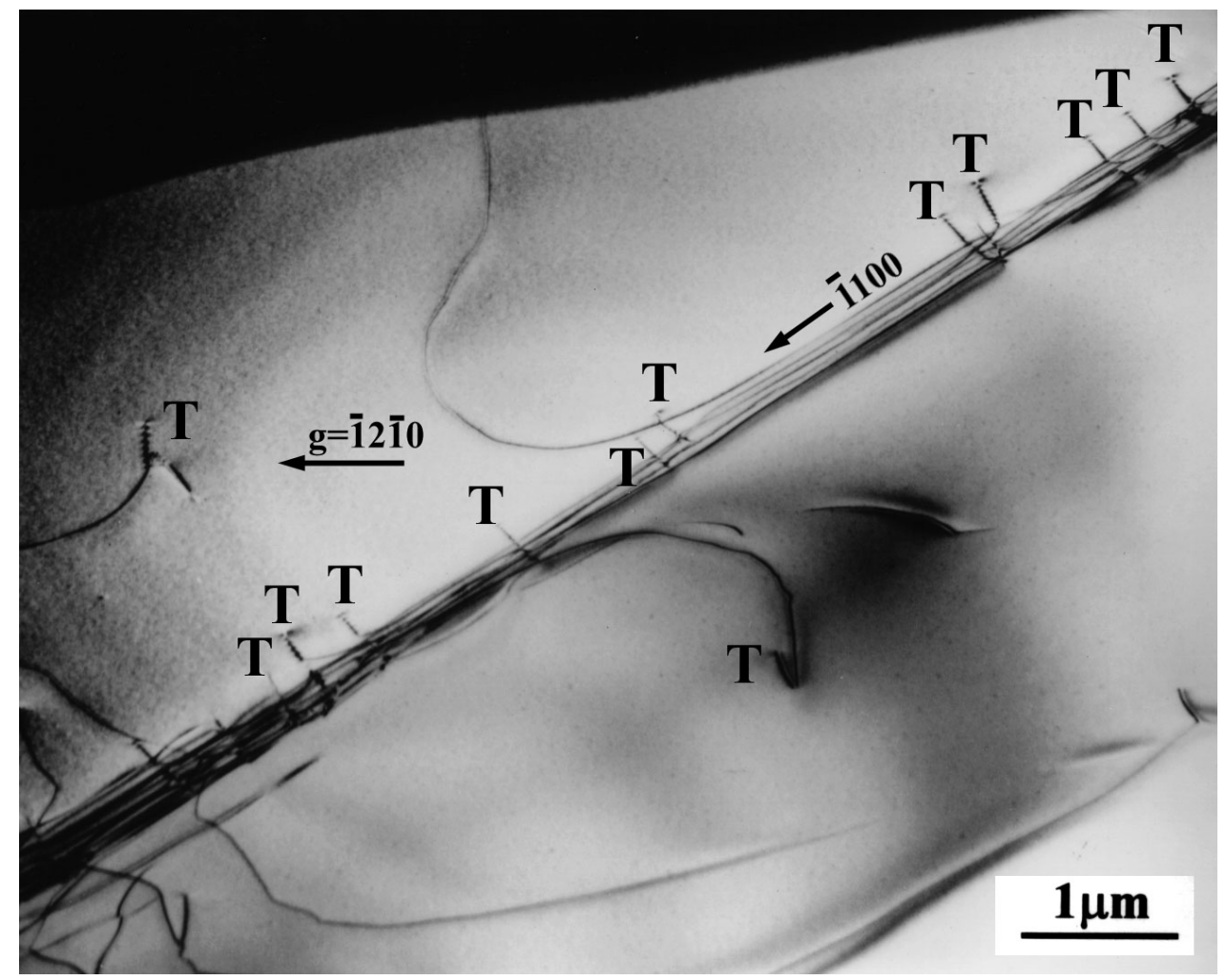

Fig.5. Z. Liliental-Weber and D. Cherns 


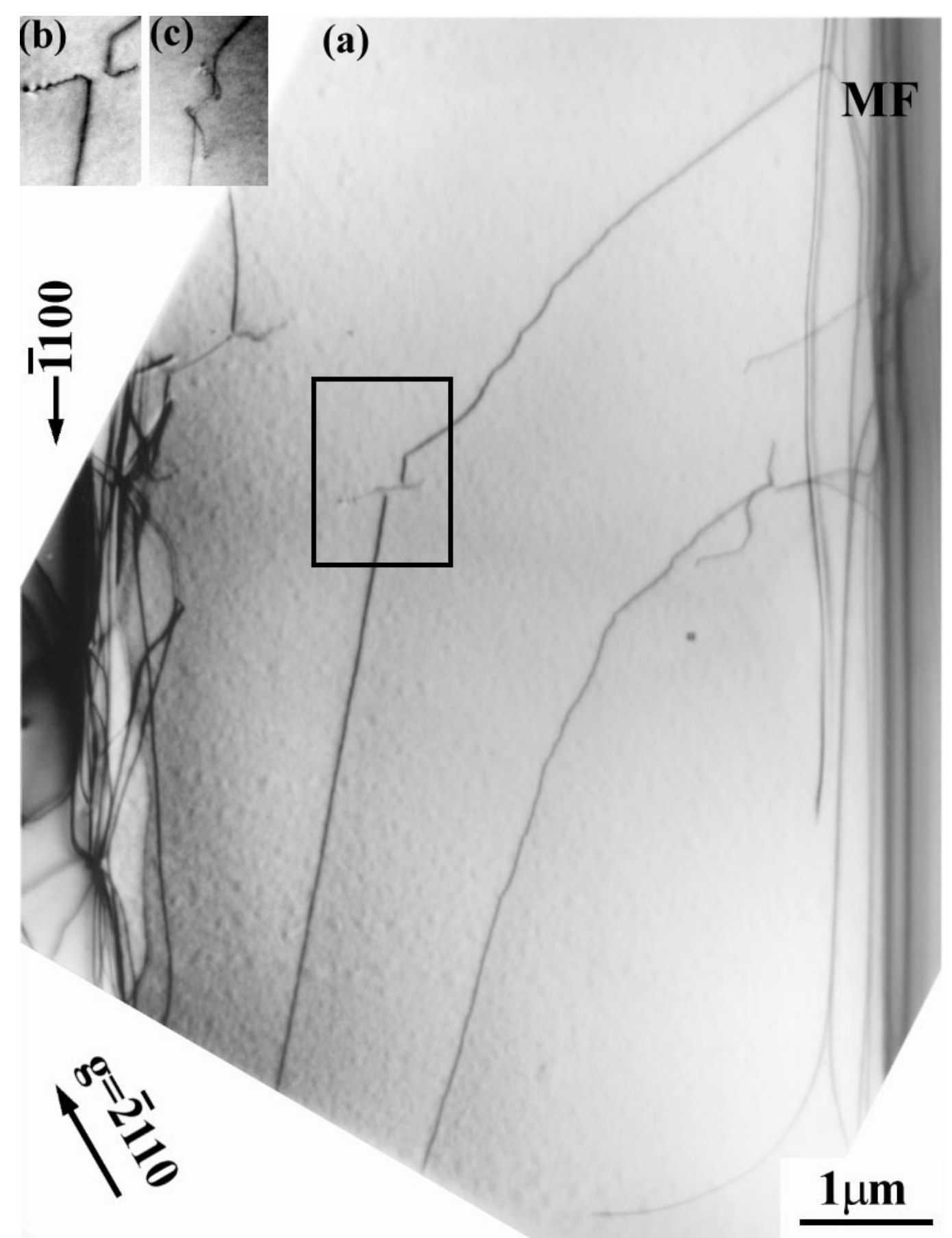

Fig.6. Z. Liliental-Weber and D. Cherns 


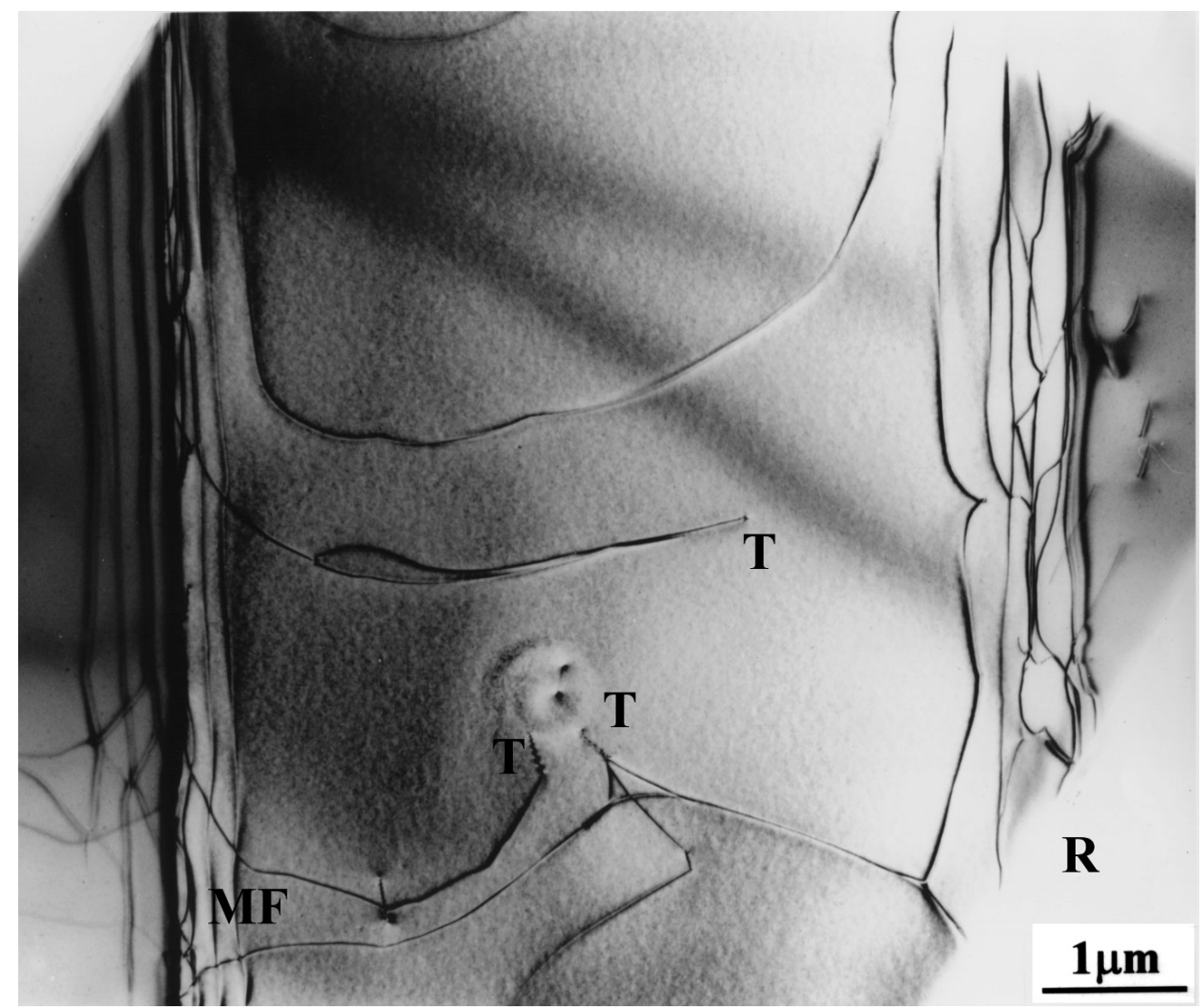

Fig.7. Z. Liliental-Weber and D. Cherns 


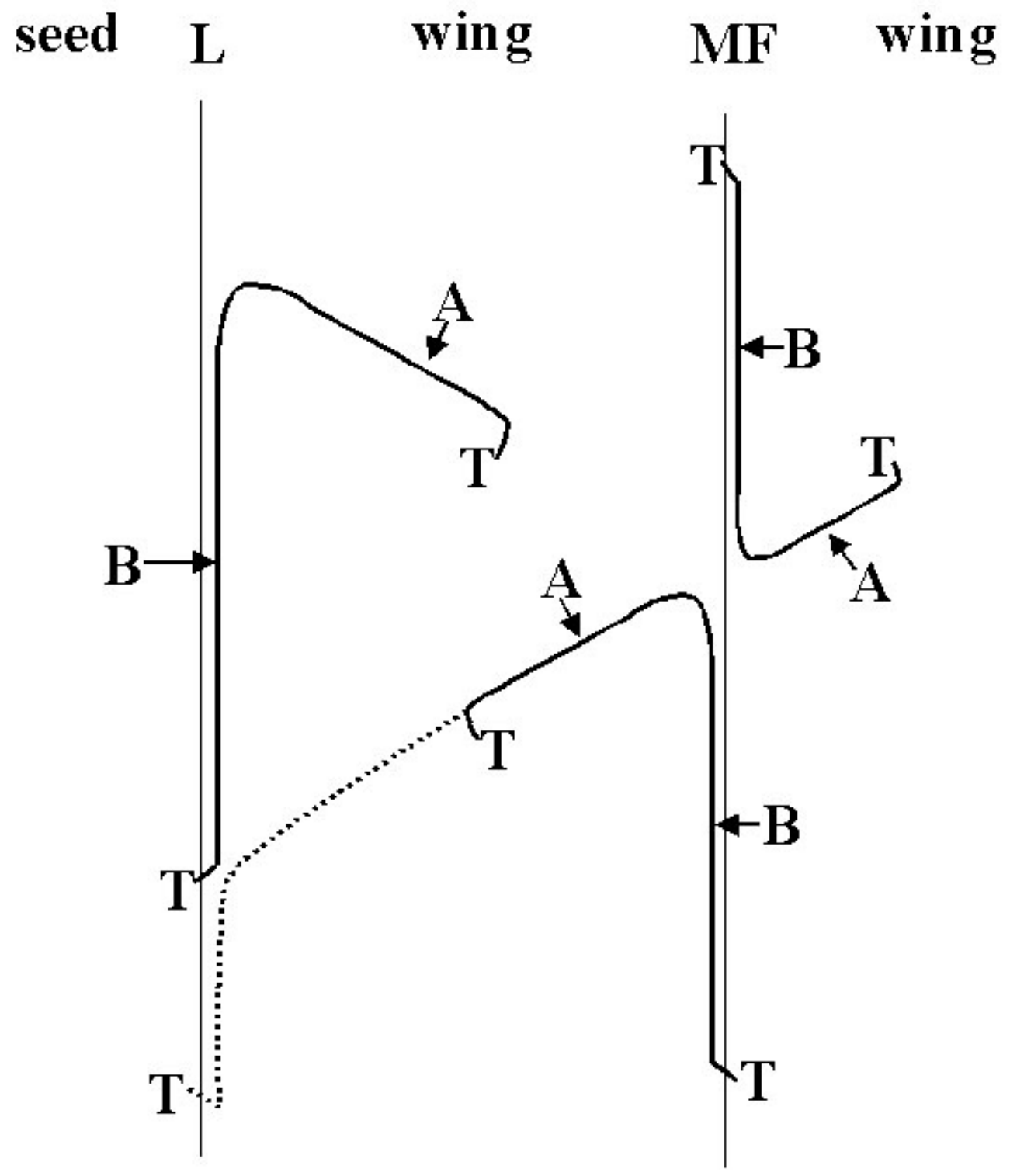

Fig.8. Z. Liliental-Weber and D. Cherns 


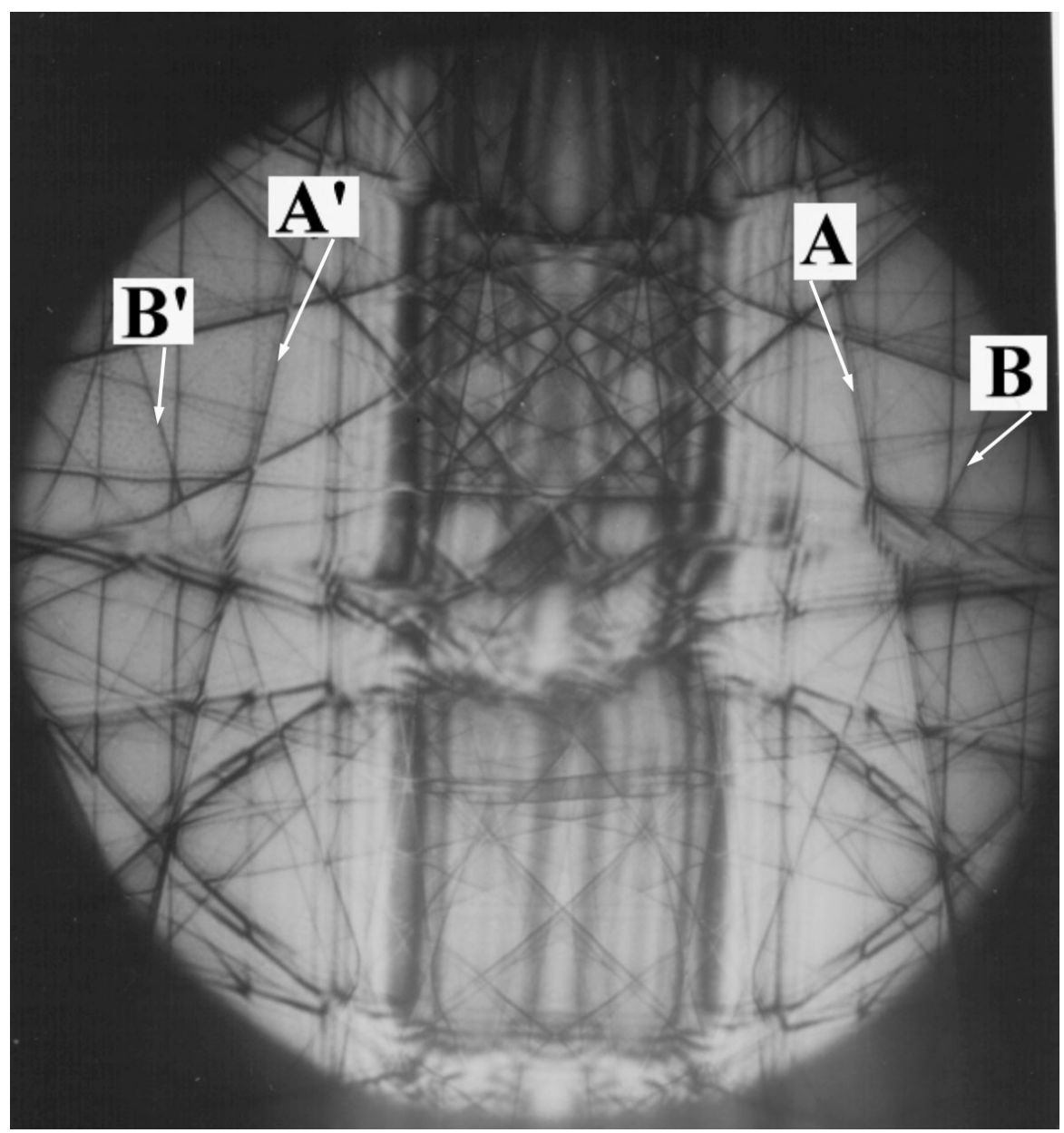

Fig.9. Z. Liliental-Weber and D. Cherns 


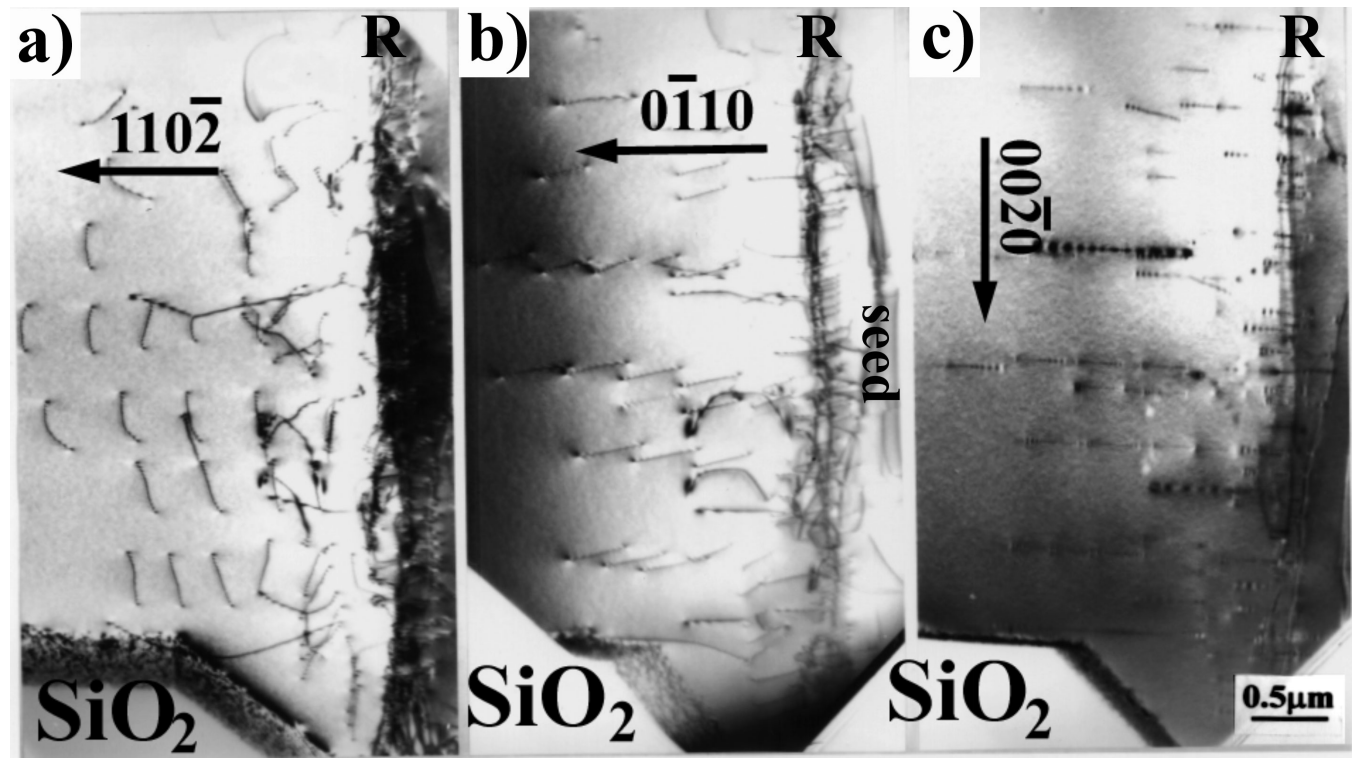

Fig.10. Z. Liliental-Weber and D. Cherns 


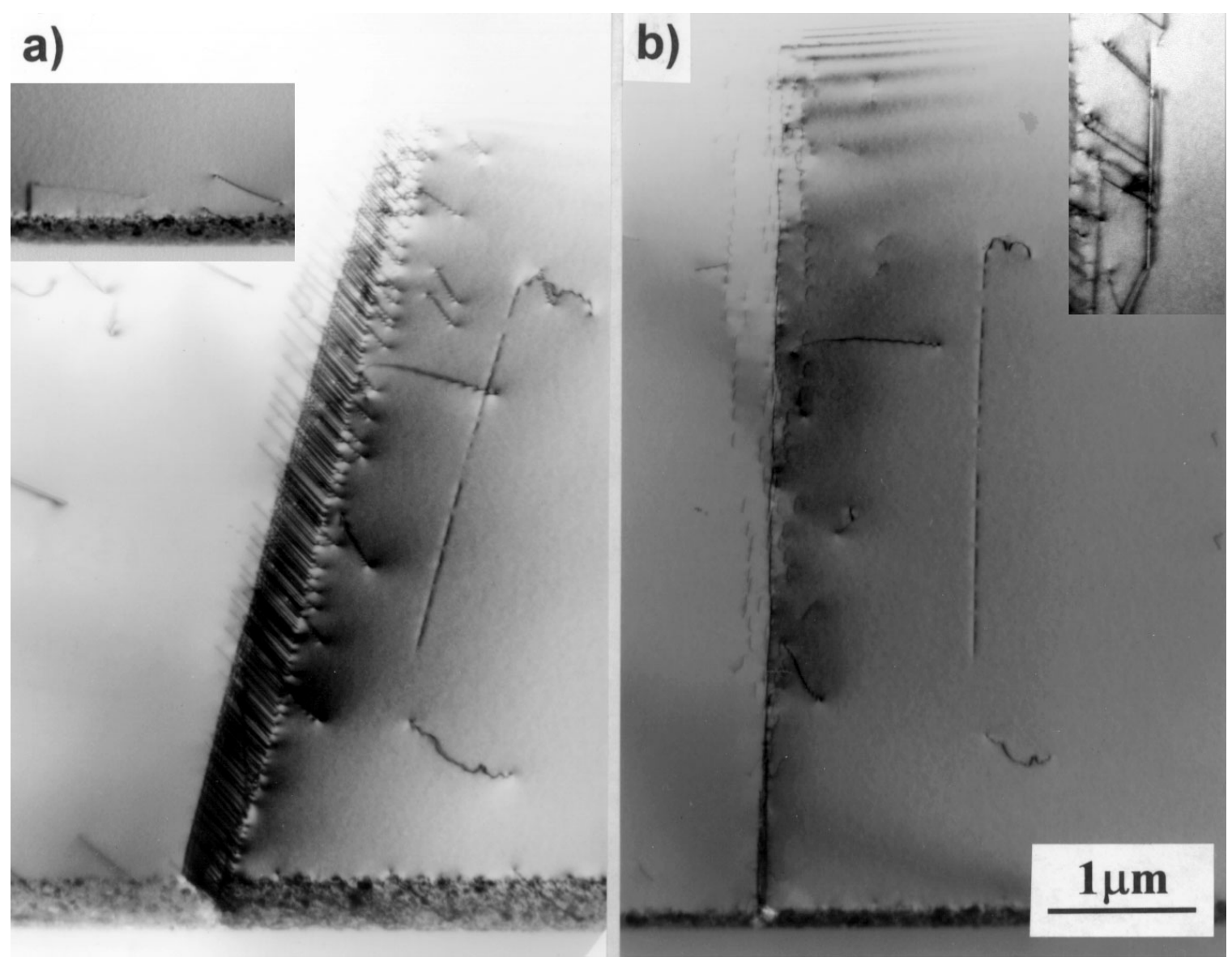

Fig.11. Z. Liliental-Weber and D. Cherns 


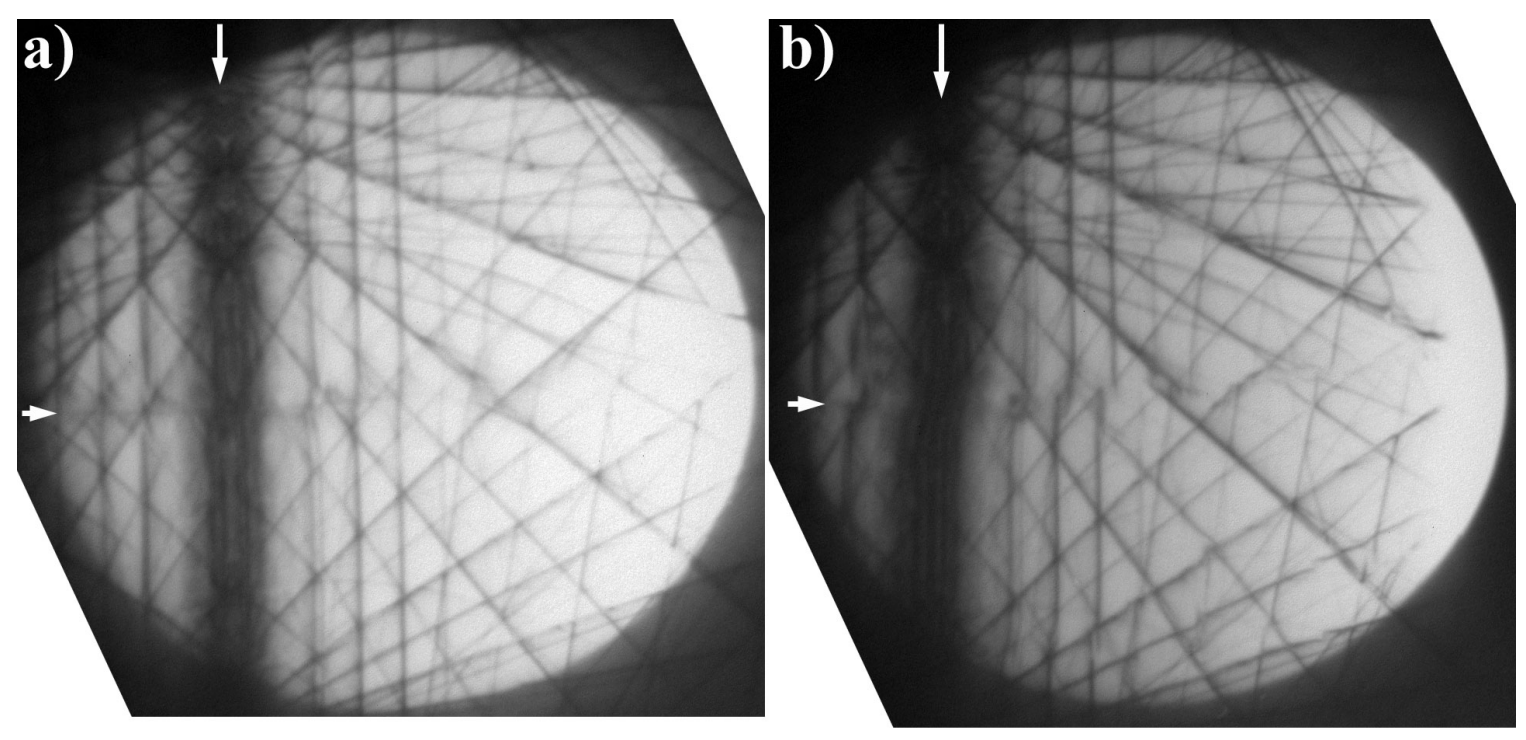

Fig.12. Z. Liliental-Weber and D. Cherns 


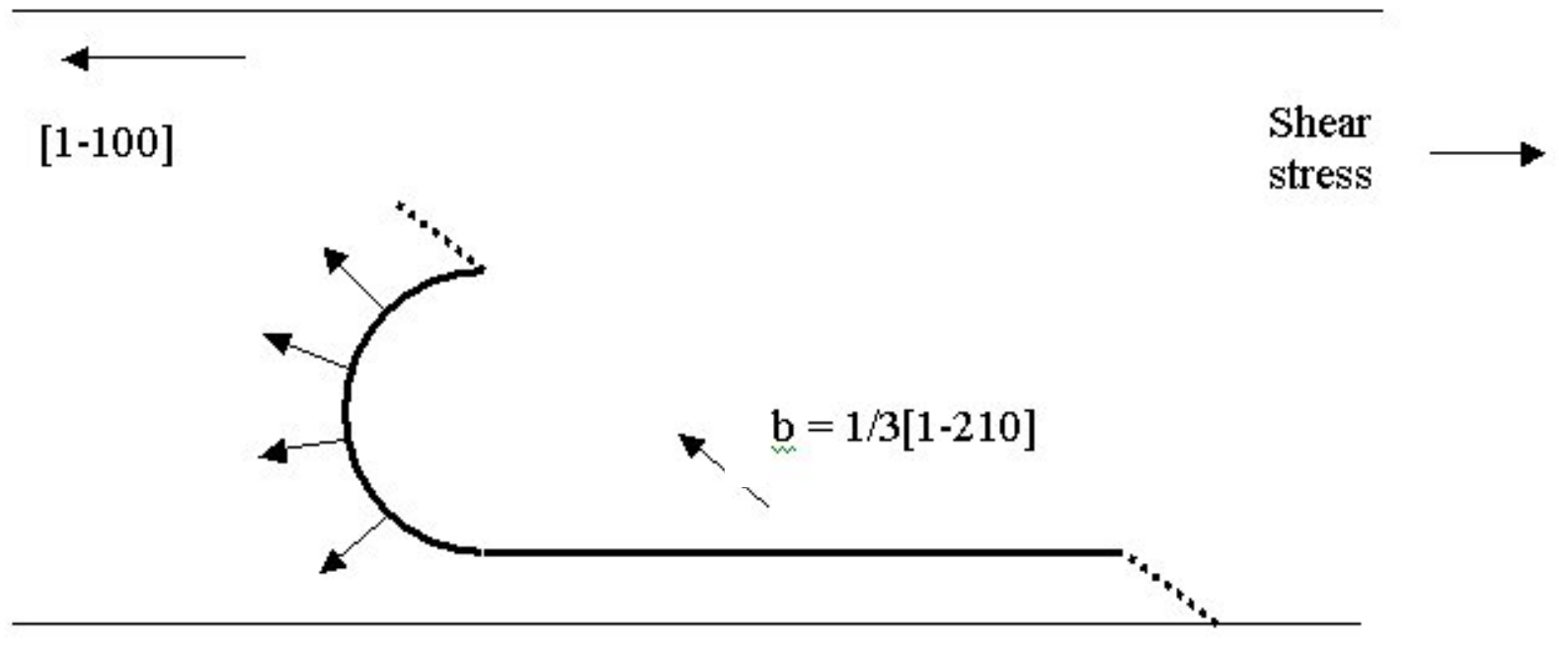

